\title{
Gerencia creativa para el desarrollo del marketing social como aprendizaje regional
}

\author{
Creative management for the \\ development of social marketing as \\ regional learning
}

\section{Wlamyr Palacios Alvarado ${ }^{1}$}

\section{Byron Medina Delgado²}

\section{Mawency Vergel Ortega ${ }^{3}$}

Universidad Francisco de Paula Santander, Cúcuta - Colombia

\section{RESUMEN}

El artículo analiza la gerencia creativa requerida para el sector industrial de Cúcuta, Colombia, dentro de la perspectiva del marketing social. La metodología se basa en el tipo de investigación documental y descriptiva, aplicada a una población. Se identificó que el sector industrial de Cúcuta dadas las calificaciones ponderadas, es más endógeno que exógeno, presenta mayor

$1 \quad$ Universidad Francisco de Paula Santander, Cúcuta, Colombia https://orcid.org/0000-0002-4292-4178

wlamyrpalacios@ufps.edu.co

2 Universidad Francisco de Paula Santander, Cúcuta, Colombia https://orcid.org/0000-0003-0754-8629 byronmedina@ufps.edu.co

3 Universidad Francisco de Paula Santander, Cúcuta, Colombia Docente - Investigadora

https://orcid.org/0000-0001-8285-2968

mawencyvergel@ufps.edu.co orientación hacia la atención de compromisos con el ambiente interno, con calificación de 3,5, que, hacia la atención de compromisos con el ambiente externo, con calificación de 2,8; requiriendo cambios en los tipos de gerencia actual para mejorar su desempeño. Finalmente, se concluye que la naturaleza de la gerencia en las industrias de Cúcuta con enfoque basado en el marketing social es creativa, exógena, holística, social, proactiva, democrática, competente y reorganizativa.

\section{PALABRAS CLAVE:}

Gerencia creativa, marketing social, sector industrial. 


\section{ABSTRACT}

The article describes the creative management required for the industrial sector of Cúcuta, Colombia, within the perspective of social marketing. The methodology is based on the type of documentary and descriptive research, applied to a population. It was identified that the industrial sector of Cúcuta, given the weighted qualifications, is more endogenous than exogenous, it presents a greater orientation towards the attention of commitments with the internal environment, with a score of 3.5 , than, towards the attention of commitments with the external environment, with a score of 2.8; requiring changes in the types of current management to improve their performance. Finally, it is concluded that the nature of management in the Cúcuta industries with a focus based on social marketing is creative, exogenous, holistic, social, proactive, democratic, competent and reorganizational.

\section{KEYWORDS:}

Creative management, social marketing, industrial sector.

\section{INTRODUCCIÓN}

Frente a la nueva economía mundial caracterizada por la globalización y el libre comercio, la gerencia de las organizaciones está obligada a innovar si desea hacer presencia en los mercados de interés, dado que la producción participa cada vez más en el comercio internacional y por lo tanto debe enfrentar a una competencia más agresiva. Dentro de este marco, surge la idea del presente artículo de proponer una gerencia creativa para el desarrollo del marketing social en la industria de la ciudad de Cúcuta, así como analizar el entorno socio-económico del sector industrial, determinar el desempeño del sector industrial desde y hacia su medio ambiente externo y hacia su medio ambiente interno y, diseñar la gerencia creativa requerida para el sector industrial dentro de la perspectiva del marketing social. El diseño metodológico utilizado define el tipo de investigación documental y descriptiva para una población de 2.238 industrias en Cúcuta que se analizan a través de una muestra de 93 establecimientos, identifica los instrumentos de recolección de información y explica el análisis de la información.

En el actual escenario, las nuevas relaciones de distribución y de producción establecen una economíainternacionalcadavezmáscompetitiva, es así que las organizaciones con el propósito de participar en los mercados de interés, deben incorporar innovaciones científicas a través de la investigación e innovaciones tecnológicas de información y comunicación que requieren una gerencia creativa y competente, para generar bienestar a la comunidad y al talento humano con responsabilidad social.

Frente a estas consideraciones, la dirección de las organizaciones debe focalizar su gestión su medio ambiente externo e interno dentro de un proceso de marketing social, enfoque definido por Kotler y Zaltman (1971): citado en Ramos y Periañez como "el marketing social es el diseño, implementación y control de programas pensados para influir en la aceptación de ideas sociales, implicando consideraciones de planificación de productos, precio, comunicación, distribución e investigación de marketing" (Ramos y Periañez. 2003, p.68). A título ilustrado, cabe señalar que el sector industrial de Cúcuta no es ajeno a esta realidad, sector que evidencia un bajo desempeño considerando la caída del PIB manufacturero del departamento de Norte de Santander que en 2010 creció un $1,8 \%$, en 2011 un 6,7 \%, en 2012 un $2 \%$, en 2013 mostró una disminución del -1,3 \% y en 2014 una caída del -2,2 \% (DANE, 2016, p.23). A la anterior situación se suma la deficiente participación del sector industrial en la población empleada en 
Cúcuta, del total de 351 mil personas empleadas en 2015, la industria vinculó 48 mil personas (DANE, 2016, p.37), resultado que equivale a una participación del $13,7 \%$ en la generación de empleo, indicador afectado por la informalidad laboral de la localidad que en 2017 es del 70,9 $\%$ siendo la más alta del país (ANDI, 2017.p.08).

Dentro de este orden de ideas, es importante resaltar que el bajo desempeño industrial de la ciudad, como ciudad ubicada en zona fronteriza, es causado principalmente por los cambios ideológicos y económicos ocurridos en Venezuela como el cierre de fronteras y la devaluación del bolívar que afecta el comercio exterior con el país vecino, incidiendo en la caída de la producción, en la inestabilidad laboral y en la calidad de vida de la población.

En lo esencial, resulta claro que el sector industrial de la región requiere de un liderazgo basado en una gerencia creativa que detecte oportunamente ciertos problemas sociales y emprenda acciones hacia la solución eficaz de esos problemas, a través de un proceso de marketing social, que eleven el nivel productivo $y$ competitivo de estas empresas con el propósito de ofrecer estabilidad laboral y mejorar la calidad de vida del talento humano y de la comunidad en general; en síntesis, es necesario de una gestión gerencial que dentro de un proceso creativo mitigue significativamente las causas del bajo desempeño industrial de Cúcuta como la falta de mayor vocación hacia la manufactura de parte de la población y cambios ideológicos y económicos en el país vecino; con el propósito de generar efectos positivos como una mayor presencia y notoriedad en los mercados de interés y un mejoramiento de la calidad de vida de la comunidad local.

\section{DISEÑO METODOLÓGICO}

La investigación documental apunta al "estudio de problemas con el propósito de ampliar y profundizar el conocimiento de su naturaleza, con apoyo, principalmente, en trabajos previos" (Universidad Pedagógica Experimental Libertador, s/f) por lo tanto, en este sentido el proyecto se fundamenta en fuentes secundarias de información como estudios, artículos, documentos, proyectos y libros, entre otros, que estén relacionados con el tema objeto del conocimiento como la gerencia creativa, el marketing social y sobre el medio ambiente de la industria de Cúcuta. Ahora bien, con el propósito de diagnosticar la situación actual del sector industrial de Cúcuta y su desempeño hacia el cumplimiento de los compromisos de responsabilidad social, se utiliza una investigación descriptiva que de acuerdo con Méndez, "en el estudio descriptivo se identifican características del universo de investigación, se señalan formas de conducta y actitudes del total de la población investigada" (Méndez, 2007, p.230), lo cual es posible con un cuestionario estructurado aplicado a una muestra de la población de industrias de Cúcuta.

La población de empresas registradas en la Cámara de Comercio de Cúcuta en diciembre de 2016 es de 26783 establecimientos, según el boletín económico Panorama Económico 2016 y proyecciones para 2017 (2016, p.10), las cuales incluyen 2238 empresas manufactureras estimadas, categoría que corresponde a la población de interés para la presente investigación.

Para la investigación se selecciona una muestra de 93 industrias de Cúcuta para mayor confiabilidad.

Como fuentes primarias fueron identificados los gerentes de las empresas de la ciudad que poseen la información necesaria para determinar el desempeño frente al cumplimiento de los compromisos sociales del sector en cuanto a su entorno y a su medio ambiente interno, con base en la aplicación de un cuestionario. Las fuentes secundarias identificadas como estudios, artículos, libros, documentos y proyectos de 
grado que poseen información relacionada con la gerencia creativa y el marketing social, las cuales se analizaron a través de la observación y la utilización de hojas de trabajo que permiten una recolección organizada de los datos.

\section{ANÁLISIS DEL SECTOR}

Situación social. El Plan de Desarrollo para Norte de Santander 2016-2019, contempla a la manufactura como una apuesta productiva para la reactivación social y económica de la región fronteriza, principalmente en los subsectores de calzado, confecciones y artesanías (Gobernación de Norte de Santander, 2016, p.16)

A título ilustrado cabe señalar que "la ubicación geográfica de Cúcuta es una ventaja para su economía y, sin embargo, los niveles de competitividad son bajos y con una relativa especialización" (Avendaño, 2012, pág. 194), como zona fronteriza está afectada por los cambios ideológicos que desde hace dos décadas están ocurriendo en la República Bolivariaba de Venezuela, dada su tendencia socialista en lo político, la salvaguarda de la balanza de pagos y la devaluación del bolívar en su economía. Se suma a lo anterior el frecuente cierre de la frontera que ha ocasionado una disminución considerable de compradores y una caída del comercio exterior; situación que ha generado un incremento de la tasa de desempleo del 12,2 \% en 2016 (DANE, 2017) y del 15,3\% entre junio y agosto de 2017 (Cubillos, 2017, p.01), conllevando a la informalidad laboral de la localidad del $70,9 \%$ en el 2017 , siendo la más alta del país (2017.p.08).

En materia de empleo, el sector industrial de Cúcuta y su área metropolitana sólo contribuye en un $13,7 \%$ a la generación de empleos directos, teniendo en cuenta que en la localidad tienen vinculación laboral en el sector productivo un total de 351.000 personas y de este total de empleados la industria vinculó 48.000 personas
(DANE, 2016, p.37). En materia de Necesidades Básicas Insatisfechas (NBI) el Plan de Desarrollo "Un Norte Pa'Lante" del Departamento Norte de Santander, señala que el porcentaje de personas en situación de pobreza en 2014, fue del 39,9\%, mientras que en 2013 fue de 39,4 $\%$ y en materia ambiental, el entorno del sector industrial de Cúcuta está caracterizado por el muy bajo Índice de Calidad Ambiental Urbana (ICAU), teniendo en cuenta que de acuerdo con el primer informe nacional de calidad ambiental urbana Cúcuta alcanzó un rango de 6,5, frente a Bucaramanga $(35,9)$, Cali $(33,0)$, Soledad $(27,0)$ y Barranquilla $(21,7)$ con ICAU bajo y ciudades como Medellín $(55,5)$, Bogotá $(50,9)$ e Ibagué $(45,2)$ con puntajes de ICAU medio (Ministerio del Ambiente y Desarrollo Sostenible, 2015, p.02) .

Es importante destacar que Cúcuta y su área metropolitana, atraviesan por una crisis social, económica y ambiental, que está generando graves consecuencias entre sectores de la sociedad, en especial en el sector industrial, lo cual requiere de soluciones creativas e innovadoras con criterio de responsabilidad social empresarial para hacer frente a una economía mundial competitiva, acompañada de acciones encaminadas al aprovechamiento de oportunidades que ofrecen los mercados locales, regionales, nacionales e internacionales dentro del marco del marketing social, con el fin de mejorara la calidad de vida y bienestar social de la población en la localidad. Finalamente, la dinámica socio-económica de la zona fronteriza colombo-venezolana se concentra en Cúcuta, Ureña y San Antonio, zona caracterizada por la baja calidad de vida de sus habitantes con altos porcentajes de necesidades básicas insatisfecha, fluctuación de las tasas de cambio, las políticas divergentes de cada país y las variaciones en el comercio local. 


\section{SITUACIÓN ECONÓMICA}

La población de 2.238 empresas manufactureras estimadas de un total de 26.783 establecimiento registrados en la Cámara de Comercio de Cúcuta según el boletín económico, Panorama Económico 2016 y proyecciones para 2017 (2016, p.10), representan una participación del $8,3 \%$ del total de empresas que han mostrado un bajo desempeño, teniendo en cuenta que de acuerdo con el Departamento Administrativo Nacional de Estadística (DANE), en el segundo trimestre de 2017 la producción disminuyó en $-4,4 \%$, las ventas $-3,4 \%$ y el personal ocupado $-3,4 \%$, respecto del segundo trimestre del año 2016, (Cubillos, 2017, p.01). Dentro de este marco, cabe señalar que la productividad del departamento de Norte de Santander registró en 2014 un crecimiento en el PIB de 4,7\% en relación con el año anterior, alcanzando una cifra de $\$ 12.529$ miles de millones a precios corrientes, según el informe de gestión 2014 de la Cámara de Comercio de Cúcuta; en 2015 el PIB del departamento fue de $\$ 12.353$ miles de millones a precios corrientes lo que refleja una caída del 1,4\% respecto del año anterior; y en 2016 el PIB aumentó un 3,3\% colocándose en $\$ 12.761$ miles de millones a precios corrientes. Es importante mencionar que el PIB departamental equivale al $1,7 \%$ del PIB nacional (DANE, 2016, p.01).

En esta materia, la productividad industrial en el departamento es deficiente, el bajo desempeño del sector industrial indica que en la región los procesos de producción manufacturera son deficientes ante la vocación comercial de la región, lo cual se evidencia en la caída del PIB manufacturero del departamento de Norte de Santander, dado que en 2010 creció un 1,8\%, en 2011 un $6,7 \%$, en 2012 un $2 \%$, en 2013 mostró una disminución del $-1,3 \%$ negativo y en 2014 una caída del -2,2 \% (DANE, 2016, p.23). Al respecto conviene destacar que en materia de productividad el sector de la manufactura contribuye en un $5,8 \%$ al PIB departamental según el Plan de Desarrollo para Norte de Santander 2016-2019 (p.16) y en materia de competitividad, la tasa de cambio (TRM) es una variable que incide en el desempeño del comercio exterior, dado que una devaluación del peso colombiano frente al dólar americano estimula las exportaciones y afecta las importaciones; en este sentido durante el trienio 2014-2016 la devaluación fue 13,3\% acumulativo anual, ver tabla 1.

Tabla 1. Indicadores de comercio exterior y PIB (millones de US\$)

\begin{tabular}{|l|r|r|r|}
\hline \multicolumn{1}{|c|}{ Conceptos } & \multicolumn{1}{c|}{$\mathbf{2 0 1 4}$} & \multicolumn{1}{c|}{$\mathbf{2 0 1 5}$} & \multicolumn{1}{c|}{$\mathbf{2 0 1 6}$} \\
\hline Tasa de cambio (\$/US) (1) & $2.392,45$ & $3.149,47$ & $3.069,17$ \\
\hline Total importaciones de Colombia (2) & $54.057,60$ & $44.889,37$ & $19.060,89$ \\
\hline Importaciones de Norte de Santander (2) & 140,44 & 88,31 & 86,63 \\
\hline Total Exportaciones de Colombia (2) & $54.856,75$ & $35.981,22$ & $31.394,31$ \\
\hline Exportaciones de Norte de Santander(2) & 272,78 & 193,96 & 120,01 \\
\hline Balanza Comercial Colombiana(2) & 799,15 & $(8.908,15)$ & $12.333,42$ \\
\hline Balanza Comercial Norte de Santander & 132,34 & 105,65 & 33,38 \\
\hline PIB total Norte de Santander (3) & 12.529 & 12.353 & 12.761 \\
\hline
\end{tabular}

Fuente: (1) Superintendencia Financiera de Colombia, Tasa Representativa del Mercado, 2016; (2) 
Dane, Información Estadística, Mincomercio, 2017; (3) Perfil Económico Norte de Santander 2017.

De acuerdo con las fuentes citadas, los resultados de las exportaciones de Norte de Santander señalan que han disminuído un $(33,7$ $\%)$ acumulativo anual, en 2014 el sector inustrial del departamento exportó un total de 272,78 millones de \$US y en 2016 la suma de 120,01 millones de \$US (DANE, 2017), exportaciones que participan en un 3,8 \% del total nacional, como se observa en la tabla anterior y la TRM del día Lunes 11 de Diciembre de 2017 fue de $3.016,18$ pesos colombianos por dólar estadounidense según el Banco de la República, 2017.

\section{RESULTADOS}

El sector industrial de Cúcuta de acuerdo con los resultadosdelcuestionarioaplicadoaunamuestra de 93 fábricas, está integrado principalmente por los subsectores metalmecánico según el 3 $\%$ de las respuestas, alimentos el $31 \%$, tejares el $15 \%$, confecciones el $42 \%$, construcción el $5 \%$ y plásticos el $4 \%$. Establecimientos que en promedio están operando con una antigüedad de 11 años. Cabe resaltar que el $21 \%$ de los encuestados no respondieron a la pregunta sobre el tipo de empresa, lo que representa un indicio de informalidad; además, el $63 \%$ declaran que poseen un nivel tecnológico bajo y el $37 \%$ medio. En materia de cobertura de mercado se detectó una deficiente cultura hacia el comercio internacional, teniendo en cuenta que sólo el $14 \%$ de las industrias locales tienen presencia en mercados internacionales, lo cual demuestra una deficiente integración con otros mercados.

De acuerdo con la Ley 905 de 2004, por medio de la cual se modifica la Ley 590 de 2000 sobre promoción del desarrollo de la micro, pequeña y mediana empresa colombiana, la industria de Cúcuta en un $91 \%$ es microempresa por tener menos de 10 empleados, el $7 \%$ es pequeña empresa por vincular entre 11 y 50 empleados $y$, un $2 \%$ es mediana empresa por vincular entre 51 y 200 empleados. En materia del grado de cumplimiento de compromisos sociales o grado de responsabilidad social empresarial se considera el umbral con calificación de 3,0 sobre 5,0 , el que determina las fortalezas o debilidades, ya sea que las respuestas por factor superen el umbral o que estén por debajo de dicho umbral respectivamente. En las Tablas 2 y 3 , se resumen los resultados del cuestionario, mostrando los logros alcanzados frente al medio ambiente externo y al medio ambiente interno.

Tabla 2. Compromisos sociales de la industria de Cúcuta con el ambiente externo

\begin{tabular}{|l|c|c|c|}
\hline \multicolumn{1}{|c|}{ Factores } & Alta & Media & Baja \\
\hline \multicolumn{1}{|c|}{ Fortalezas } & $\mathbf{5 , 0}-\mathbf{4 , 5}$ & $\mathbf{4 , 4}-\mathbf{3 , 5}$ & $\mathbf{3 , 4}-\mathbf{3 , 0}$ \\
\hline Relación con los medios de comunicación & 4,9 & & \\
\hline Cuentan con plan de manejo ambiental & & & 3,2 \\
\hline \multicolumn{1}{|c|}{ Debilidades } & $\mathbf{1 , 0}-\mathbf{1 , 9}$ & $\mathbf{2 , 0}-\mathbf{2 , 4}$ & $\mathbf{2 , 5 - 2 , 9}$ \\
\hline Programas hacia la comunidad & & 2,0 & \\
\hline Relación con familias de trabajadores & & & 2,8 \\
\hline Relación con instituciones educativas & & & 2,6 \\
\hline Relación con los gremios & & & 2,9 \\
\hline Realizan obras de mejoras para la ciudad & 1,6 & & \\
\hline Realizan aportes a la población vulnerable & & 2,3 & \\
\hline
\end{tabular}


Tabla 3. Compromisos sociales de la industria de Cúcuta con el ambiente interno

\begin{tabular}{|l|c|c|c|}
\hline \multicolumn{1}{|c|}{ Factores } & Alta & Media & Baja \\
\hline \multicolumn{1}{|c|}{ Fortalezas } & $\mathbf{5 , 0}-\mathbf{4 , 5}$ & $\mathbf{4 , 4}-\mathbf{3 , 5}$ & $\mathbf{3 , 4}-\mathbf{3 , 0}$ \\
\hline Programas de formación al talento humano & & & 3,2 \\
\hline Servicios sociales al empleado & & & 3,3 \\
\hline Cumplen con legislación laboral & 5,0 & & \\
\hline Programas de vivienda & & & 3,4 \\
\hline Programas de recreación & 4,5 & & \\
\hline \multicolumn{1}{|c|}{ Debilidades } & $\mathbf{1 , 0}-\mathbf{1 , 9}$ & $\mathbf{2 , 0}-\mathbf{2 , 4}$ & $\mathbf{2 , 5 - 2 , 9}$ \\
\hline Fondo de empleados & & $\mathbf{2 , 1}$ & \\
\hline
\end{tabular}

Finalmente, el sector industrial de Cúcuta dadas las calificaciones ponderadas, es más endógeno que exógeno, presenta mayor orientación hacia la atención de compromisos con el ambiente interno $(3,5)$, que hacia la atención de compromisos con el ambiente externo $(2,8)$.

\section{NATURALEZA DEL CARGO.}

Direccionar a las organizaciones hacia un proceso que detecten oportunamente ciertos problemas sociales y planifiquen y ejecuten acciones para la solución eficaz de esos problemas, en otras palabras, que implementen un modelo de marketing social, requieren de un estilo gerencial cuya naturaleza puede resumirse en una sola palabra, creatividad. Según la definición de Tudor Powell sobre la creatividad, citado en Hernández, corresponde a "una combinación de flexibilidad, originalidad y sensibilidad orientada hacia ideas que permiten a la persona creativa desprenderse de las secuencias comunes de pensamiento y producir otras secuencias de pensamiento, diferentes y productivas, cuyo resultado ocasiona satisfacción a ella misma y tal vez a otros" (Hernández, 2011, p.11); es decir, pasar de una orientación con énfasis económico a una orientación con énfasis social, más exógena que endógena, más centrada en el entorno que en los aspectos internos para detectar oportunidades y necesidades insatisfechas y con ello tomar decisiones pertinentes encaminadas a la solución de problemas. Al respecto, el sector industrial de Cúcuta debe implementar un modelo de responsabilidad social empresarial para cumplir con los compromisos sociales, dado que de acuerdo con la investigación, el desempeño en esta materia es deficiente en cuanto a la gestión hacia el entorno.

Las reflexiones anteriores tienen implicados procesos holísticos mediante la interdependencias de lo externo con lo interno, elementos claves en la naturaleza de la gerencia creativa dado que permite formular las relaciones entre las variables que participan en la problemática empresarial, en este sentido, el desarrollo de la creatividad en los gerentes de las empresas industriales de Cúcuta implica la toma de decisiones en cuanto a qué hacer y qué explorar, abiertos al cambio de enfoques y puntos de vista, capaces de aplicar el conocimientos o experiencias; lo cual involucra una actitud proactiva, parte de su naturaleza que le permita afrontar los riesgos que se corren cuando se está proponiendo un nuevo paradigma, diferente al tradicional o rutinario en la solución de conflictos.

Debe señalarse que frente al desafío anterior, la mente creativa juega un papel fundamental dado que "es capaz de conectarse con sus sentidos en todos los niveles, y ello le permite, entre otras cosas, percibir los cambios que sufren el medio ambiente y el contexto inmediato" (Hernández, 
2011, p.12), es así que ser creativo, pensar creativamente o tener una actitud creativa, implica generar nuevas técnicas que permitan a los gerentes la adaptación del medio a las necesidades, para dar una respuesta a una situación en concreto, en especial a los conflixtos sociales como el alto desempleo en la localidad e informalidad laboral y, en lo económico la baja productividad en la región y caída del comercio internacional, factores que afectan al sector industrial de Cúcuta.

A título ilustrado, merece resaltar que las universidades, como espacios para la producción del conocimiento deben enfrentar las presiones políticas, sociales, económicas y culturales, es así que "la sociedad espera que estas instituciones respondan a tales presiones de forma creativa e innovadora con la participación de todos los miembros de la comunidad universitaria, especialmente de sus directivos o gerentes, a quienes les corresponde tomar iniciativa para el cambio y transformación de la organización" (Martínez, 2013, p.120). En este propósito, la integración de instituciones educativas-empresas es fundamental, aspecto que según la investigación es una debilidad en el sector industrial de Cúcuta.

Dentro de este marco, cabe considerar, que la gerencia creativa implica "la participación de todos en un clima democrático, capacidad para diagnosticar, investigar, la formación de equipos de trabajo, promover proyectos, resolver problemas, reflexiones permanentes sobre los procedimientos, las situaciones, tomando en cuenta las necesidades de todo el personal y la comunidad, con una visión prospectiva, proactiva, eficiente y eficaz, compartida con todos los actores de la organización" (Martínez, 2013, p.137).

Por otro lado, se plantea que la verdadera gerencia creativa está íntimamente relacionada con la reingeniería; es decir con la reorganización de sus procesos, esto concuerda con lo planteado por Morris y Brandon (1994) y Soto (1998), citado en Martínez, que la reingeniería es el "rediseño radical de los procesos de trabajo e implementación de nuevos diseños que permitan aumentar la capacidad para competir en el mercado; en ese análisis y reflexión del rediseño de los procesos está presente la creatividad cuando se redescubre y rediseña las reglas, se discuten las ideas y sugerencias dentro del contexto de una nueva misión y de las tecnologías de información con que cuenta la organización" (Martínez, 2013, p.138); es decir, la reingeniería que debe iniciar la industria local está centrada en investigación del mercado de interés, mejorar las relaciones con los medios de comunicación e implementación de tecnologías de la información y comunicación, mejorar las relaciones con los gremios y fomentar la formación de pequeñas cadenas productivas por subsectores industriales, implementar programas de capacitación del talento humano según necesidades, implementar programas de integración con las familias de los trabajadores y diseñar y ejecutar programas de manejo ambiental (PMA). Finalmente, la naturaleza de la gerencia en las industrias con enfoque basado en el marketing social puede resumirse en los siguientes conceptos: creativa, exógena, holística, social, proactiva, democrática, competente y reorganizativa.

\section{RESPONSABILIDADES Y FUNCIONES DE LA GERENCIA CREATIVA}

La responsabilidad de una gerencia creativa que oriente el marketing social en el sector industrial de Cúcuta, está centrada en alcanzar óptimos niveles de productividad y competitividad que generen valor al cliente, al talento humano y a la comunidad en general. En este contexto, cabe señalar que la productividad es "un indicador relativo que mide la capacidad de un factor productivo, o varios, para crear determinados bienes, por lo que al incrementarla se logran mejores resultados, considerando los recursos 
empleados para generarlos" (Miranda, 2010, p.248); esta productividad se manifiesta en la óptima utilización de los insumos al lograr un máximo de cantidades fabricadas con un mínimo de materias primas; en las economías de escala logrando mayores cantidades producidas que reducen los costo totales; en lo financiero al asociar un óptimo capital de trabajo que cubra las necesidades operativas de fondos y en la productividad en el trabajo al asignar el número óptimo de personal en cada etapa del proceso productivo.

Respecto de la competitividad es importante considerarla en dos dimensiones, la interna y la externa; la competitividad interna se relaciona con la productividad y se refiere a la "capacidad de la organización para lograr el máximo rendimiento de los recursos disponibles, como personal, capital, materiales, ideas y los procesos de transformación" (Omaña. 2012, p.82) y la competitividad externa está orientada a la consecución de "los logros de la organización en el contexto de mercado o el sector a que pertenece. Como el sistema de modelo es ajeno a la empresa, ésta debe considerar variables exógenas, como el grado de innovación, el dinamismo de la industria, la estabilidad económica, para estimar si la competitividad es a largo plazo" (Omaña. 2012, p.82); en este sentido, consiste en hacer presencia en los mercados de interes, posicionarse y sostenerse en esos mercados.

Las funciones de la gerencia pueden resumirse en el cumplimiento del proceso administrativo de planeación, dirección, organización y control, de la siguiente manera: (i) identificar las oportunidades en el entorno y las necesidades sociales en los mercados de interés; (ii) fijar los objetivos estratégicos para alcanzar óptimos niveles productivos y competitivos; (iii) planificar la operatividad de la empresa en asuntos de mercadeo, administración, organización, producción y finanzas; (iv) socializar resultados de la planeación con los jefes de área con el fin de tomar decisiones acertadas para el desarrollo de proyectos estratégicos y acciones a seguir; (v) contratar y capacitar al personal requerido en cada área clave del éxito y en todos los niveles de la organización; (vi) diseñar, evaluar y aprobar el presupuesto global de la empresa y asignar los recursos financieros necesarios para la adecuada operatividad; (vii) evaluar los resultados logrados en cada mes y efectuar comparativos contra los planes para evitar desviaciones; (viii) desarrollar proyectos estratégicos que generen valor agregado al cliente externo e interno y a la comunidad en general; (ix) diseñar, ordenar y evaluar semestralmente el balance social de la empresa en cuanto al cumplimiento de los compromisos con el entorno y con lo interno; (x) determinar la participación en las mejoras de la ciudad y asignar recursos para ello; (xi) mantener en alto el nivel de satisfacción del cliente externo, interno y de la comunidad en general; (xii) ejecutar programas de formación y capacitación del talento humano en los temas pertinentes a la empresa; (xiii) analizar y apoyar las iniciativas creativas e innovadoras del talento humano que apunten a la integración con la comunidad y al mejoramiento del proceso productivo; y (xiv) diseñar el plan de manejo ambiental y velar por su cumplimiento.

\section{EFECTOS DEL MARKETING SOCIAL EN LA INDUSTRIA DE CÚCUTA}

Toda organizacón requiere de la aceptación social para asegurar la preferencia en un mercado y su imagen favorable ante los consumidores mediante la solución de problemas sociales a través de un proyecto de marketing social que marca la orientación basada en la investigación sobre los deseos, gustos, preferencias, creencias y actitudes específicas de los consumidores, así como de las características de los productos sociales que pretenden fabricarse y ofrecerce, orientación que surte efectos positivos en lo 
externo y en lo interno. En lo esencial, el efecto más directo del mercadeo social está dado en la relación de intercambio establecida entre la empresa y organizaciones sociales, o entre la empresa y sus clientes, a través de productos con valor agregado para que los clientes se sientan satisfechos y así la empresa logre el efecto de la notoriedad y el reconocimiento e incremento en la producción y en su volumen de ventas; dado que una opinión pública favorable, en términos de mercadeo social, influye en la decisión de compra del consumidor. En este sentido, el sector industrial de Cúcuta es percibido según la investigación, con muy buena relación con los medios de comunicación, sin embargo, las relaciones con las familias de los empleados, con los gremios, con las instituciones educativas y programas hacia la comunidad son considerados como debilidades, es por ello que los efectos en la notoriedad y reconocimiento e incremento en la producción y en su volumen de ventas no son satisfactorios.

Frente a la situación anterior, resulta claro que para generar efectos positivos en los mercados de interés, el sector industrial de Cúcuta debe implementar programas de responsabilidad social empresarial (RSE), concepto definido por World Business Council for Sustainable Development (2008) como "compromiso que asume una empresa para contribuir al desarrollo económico sostenible por medio de colaboración con sus empleados, sus familias, la comunidad local y la sociedad, con el objeto de mejorar la calidad de vida" (Rendueles, 2010, p.36).

En este marco, surge entonces la necesidad de un balance social "como instrumento que permita reportar resultados en términos de inversiones sociales llevadas a cabo para un período determinado, con el fin de comunicar e informar a accionistas, empleados y a toda la sociedad la aplicación de recursos destinados a cumplir con las responsabilidades sociales pautadas por la organización" (Rendueles, 2010, p.36); este instrumento debe ser diligenciado por las empresas del sector industrial de Cúcuta semestralmente y, la evaluación de resultados mediante indicadores debe conducir a mejoras en el cumplimiento de los compromisos sociales en los ámbitos externo e interno.

De acuerdo con lo anterior, es posible señalar que el sector industrial de Cúcuta de acuerdo con los resultados de la investigción, presenta mayor énfasis hacia los compromisos internos $(3,5)$ que hacia la atención de compromisos con el entorno $(2,8)$; por lo tanto, para mejorar estos resultados debe implementarse el balance social para superar la calificación por indicador mediante evaluaciones semestrales, dado que es la herramienta de medición de la gestión social de las organizaciones.

Los efectos positivos que el marketing social genera, se evidencian a través de una gerencia creativa en la industria de Cúcuta, que apunte su gestión hacia las siguientes acciones en el ámbito externo: (i) programas de integración con las familias de los trabajadores a través de eventos en épocas especiales; (ii) programas conjuntos con colegios, universidades y demás instituciones educativas para el ofrecimiento de formación y capacitación del talento humano en temas pertinentes a cada industria; (iii) desarrollo de programas con los gremios como Acopi y Andi para la formación y el fortalecimiento de pequeñas cadenas productivas por subsectores; (iv) obras en la ciudad como mantenimiento de vías arterias, monumentos históricos, bibliotecas, centros culturales y galerías de arte entre otros; (v) desarrollo de proyectos comunitarios como financiamientos de escuelas y colegios para población vulnerable, programas de reforestación y cursos sobre sobre preservación ambiental; y (vi) programas de divulgación de proyectos sociales a través de los medios de comunicación. 


\section{CONCLUSIONES}

Basados en el análisis documental y el análisis del instrumento aplicado se conluyen las siguientes afirmaciones:

La industria es una apuesta productiva para la reactivación social y económica de la región fronteriza de Norte de Santander y su capital Cúcuta contemplada en el Plan de Desarrollo para Norte de Santander 2016-2019.

El principal problema social que está afectando la calidad de vida de la población en Cúcuta es el desempleo, dado que en 2017 se ubicó en $15,3 \%$, situación que a su vez conlleva a la informalidad laboral de la localidad que en ese año es del 70,9 \% siendo la más alta del país.

El porcentaje de personas en situación de pobreza en 2014 , fue de $39,9 \%$, lo que señala un alto índice en materia de necesidades básicas insatisfechas, que afecta la calidad de vida de las familias de Cúcuta. El sector industrial de Cúcuta participa en un $8,3 \%$ del total de empresas, sector que ha mostrado un bajo desempeño teniendo en cuenta que en el segundo trimestre de 2017 la producción disminuyó $-4,4 \%$, las ventas $-3,4 \%$ y el personal ocupado $-3,4 \%$, respecto del segundo trimestre del año 2016.

El sector industrial de Cúcuta, de acuerdo con los resultados del cuestionario aplicado a una muestra de 93 fábricas, está integrado principalmente por los subsectores metalmecánico según el $3 \%$ de las respuestas, alimentos el $31 \%$, tejares el $15 \%$, confecciones el $42 \%$, construcción el $5 \%$ y plásticos el 4 $\%$; establecimientos que en promedio están operando con una antigüedad de 11 años.

Se percibe una tasa de informalidad empresarial del $21 \%$ en el sector industrial de Cúcuta, quienes operan con un nivel tecnológico bajo en un $63 \%$ y un nivel tecnológico medio en un 37 $\%$.
Se detectó una deficiente cultura del sector hacia el comercio internacional, teniendo en cuenta que sólo el $14 \%$ de las industrias locales tienen presencia en mercados internacionales, lo cual demuestra una deficiente integración con otros mercados.

La industria de Cúcuta en un $91 \%$ es microempresa por tener menos de 10 empleados, el $7 \%$ es pequeña empresa por vincular entre 11 y 50 empleados y un $2 \%$ es mediana empresa por vincular entre 51 y 200 empleados, de acuerdo con la Ley 905 de 2004, por medio de la cual se modifica la Ley 590 de 2000 sobre promoción del desarrollo de la micro, pequeña y mediana empresa colombiana.

El sector industrial de Cúcuta es más endógeno que exógeno, presenta mayor orientación hacia la atención de compromisos con el medio ambiente interno $(3,5)$ que hacia la atención de compromisos con el medio ambiente externo $(2,8)$.

La naturaleza de la gerencia en las industrias de Cúcuta con enfoque basado en el marketing social es creativa, exógena, holística, social, proactiva, democrática, competente y reorganizativa.

Las funciones de la gerencia creativa en general están dadas a través del cumplimiento del proceso administrativo de planeación, dirección, organización y control.

Una gerencia creativa que direccione el marketing social en el sector industrial de Cúcuta genera efectos e impactos en el desarrollo sostenible del sector, teniendo en cuenta que: en lo social eleva el nivel de satisfacción del empleado y de la comunidad en general y, en el mejoramiento de la calidad de vida de las familias; en lo económico al incrementar el nivel productivo y competitivo de las industrias; y en lo ambiental al conservar el medio ambiente y los recursos naturales. 
La responsabilidad social empresarial debe basar la gestión en la gerencia creativa dado que es una excelente herramienta para lograr notoriedad, posicionamiento y sostenimiento en los mercados de interés; además, es una estrategia del mercadeo social para fortalecer la imagen de la industria como organización socialmente responsable.

\section{REFERENCIAS BIBLIOGRÁFICAS}

ANDI. Asociación nacional de Industriales, Informe de Vicepresidencia de desarrollo económico y competitividad, Bogotá. Año 2017, Pag.08.

ARISTIZÁBAL, Carlos A. LEÓN, Mario. ARIAS, José. Impactos del mercadeo social en organizaciones de servicios. Revista semestre económico. Vol.10, Año 2017, No.19.

BANCO DE LA REPÚBLICA. Tasa de Cambio del peso colombiano (TRM), Bogotá. Año 2017.

BIBLIOTECA DEL LABORATORIO. Mettler T. Glosario de Términos de Mercadeo, (año 2017). Recuperado de: http://www.mercadeo.com/ glosario.htmA

BOLETÍN ECONÓMICO. Panorama Económico 3016 y proyecciones para 2017. Año 2017. Recuperado de: http://www.cccucuta.org. co/media/Adjuntos_de_Noticias/informe economico.pd

CÁMARA DE COMERCIO. Informe de Gestión, San Jose de Cúcuta, Año 2014.

CÓRDOBA, José F. Del marketing transaccional al marketing relacional. Revista Entramado. Vol. 5, Año 2009, No. 1, Pág. 6-17.

CRISSIEN, John, O. Gerencia del Siglo XXI, Revista Escuela de Administración de Negocios. Año 2005, No. 54, Pág. 59-83.
CUBILLOS, Natalia. Cúcuta espera renacer económico con cuero, calzado y confecciones, San José de Cúcuta. Año 2017. Recuperado de: http://www.elcolombiano.com/negocios/cucutaespera-renacer-economico-con-cuero-calzado$y$-confecciones-DE7521036

CURIEL Lorenzo, S. Elementos de una campaña de marketing social para la promoción de la mediateca municipal de Soyo, Angola. Revista de Arquitectura e Ingeniería. Vol. 6, Año 2012, No.3, Pág. 1-8.

DANE. Departamento administrativo nacional de estadística, Información Estratégica. Indicares Económicos, Bogotá. Año 2016.

DANE. Departamento administrativo nacional de estadística, Información Estadística, Mincomercio, Perfil Económico Norte de Santander, Bogotá, Año 2017.

GIULIANO Antonio C. Monteiro, Thel A. Zambon, Marcelo S. Betanho C. El Marketing Social, el Marketing relacionado con causas sociales y la Responsabilidad Social Empresarial, Caso del Supermercado Pão De Açúcar, de Brasil Invenio. Revista Invenio. Vol. 15, Año 2012, No. 29, Pág. 11-27

GOBERNACIÓN DE NORTE DE SANTANDER, Plan de Desarrollo para Norte de Santander 2016-2019 "Un Norte Productivo Para Todos", Cúcuta. Año 2016.

GÓNGORA Ledia H. Mercadotecnia social: una herramienta necesaria para la promoción de salud. Revista Medisan. Vol. 18. Año 2014. No. 5. Pág. 691-702.

HERNÁNDEZ Raquel, Creatividad y Actitud Creativa. Revista del Centro de Investigación. Vol. 9, Año 2011, Pag. 11- 15.

JANY, José N. Investigación integral de mercados. Editorial McGraw Hill, Bogotá. Año 2000. 
MARTÍNEZ, Sol A. Gerencia creativa en el Instituto Pedagógico de Caracas. Revista de Investigación. Vol. 37, Año 2013, No. 79, Pág. 119-143.

MÉNDEZ, Carlos. Metodología. Limusa Editores, Bogotá. Año 2007.

\section{MINISTERIO DEL AMBIENTE Y DESARROLLO} SOSTENIBLE. Minambiente revela cifras del estado ambiental de 128 áreas urbanas de Colombia, Bogotá. Año 2015.

MIRANDA, Jorge.Toirac, Luis. Indicadores de Productividad para la Industria Dominicana. Revista Ciencia y Sociedad. Vol. XXXV, Año 2010, No. 2, Pag. 235-290.

RAMOS, João A. Periáñez, Iñaki. Delimitación del Marketing con Causa o Marketing Social Corporativo mediante el análisis de empresas que realizan acciones de responsabilidad social. Vol. 03, Año 2003, No. 1-2, Pág. 65-82.
RENDUELEs, Miguel. Mercadeo social, responsabilidad social y balance social: conceptos a desarrollar por instituciones universitarias. Revista Telos. Vol. 12, Año 2010. No.1, Pag. 29-42.

OMAÑA, Carol del Valle. Pensamiento creativo para la generación de competitividad en las organizaciones educativas. Observatorio Laboral Revista Venezolana. Vol. 5, núm. 10, Año 2012, Pag. 79-99.

SERCA CORPORATIVO. ¿Qué es un estudio socioeconómico, cuál es el contenido y que tipos de socioeconómicos existen? Año 2013. Recuperado de:http://corporativoserca.com/ blog/que-es-un-estudio-socioeconomicocual-es-el-contenido-y-que-tipos-desocioeconomicos-existen/

SUPERINTENDENCIA FINANCIERA DE COLOMBIA. Tasa Representativa del Mercado. Año 2016. Recuperado de: https://www. superfinanciera.gov.co/jsp/index.jsf 